\title{
EXACT EMBEDDINGS INTO ARTINIAN RINGS
}

\author{
RAYMOND A. BEAULIEU AND ANDERS JENSEN
}

(Communicated by Maurice Auslander)

\begin{abstract}
The concept of exact embedding of a Noetherian ring into an Artinian ring discussed by Blair and Small is exploited to yield results on embeddings of various rings into Artinian rings. In particular we investigate Ore extensions, group graded rings, and filtered rings. Also, we prove that exact embeddability is a Morita invariant.
\end{abstract}

\section{INTRODUCTION}

In recent years there has been considerable interest in determining when (right) Noetherian rings embed in Artinian rings. Goldie's and Small's theorems characterize those rings with (semi-) simple Artinian and Artinian classical quotient rings. The somewhat weaker question asking only when a ring embeds in an Artinian ring has been studied by several authors. Small [17], and later, Dean [6] and Dean-Stafford [7] gave examples of one- and two-sided Noetherian rings failing to embed in Artinian rings. On the other hand, a classification of homomorphisms from a right Noetherian $k$-algebra to simple Artinian rings, due to Schofield [15], was used by Blair-Small (see [3, 4]) to establish the embeddability of, for example, Krull-homogeneous algebras and affine PI-algebras. Inherent in $[3,4]$ is the concept of exact embedding, the study of which is the subject of this paper.

\section{CONVENTIONS AND BACKGROUND}

In what follows we adopt the convention that Noetherian (Artinian) means two-sided Noetherian (Artinian), i.e., when chain conditions are assumed on one side only we will indicate this explicitly. All rings considered have 1 and homomorphisms take 1 to 1 . The prime radical of a ring $R$ is denoted $N(R)$, and for an ideal $I \triangleleft R$ we set $\mathscr{C}_{R}(I)=\{a \in R \mid \bar{a}$ is regular in $R / I\}$. DCC is short for descending chain condition. All modules are considered to be right modules unless explicitly stated otherwise, and $k$ denotes an arbitrary field.

For general background on Sylvester rank functions and Schofield's results we refer to $[3,15,12]$. Here we only state the results that we will actually need in the sequel.

Received by the editors June 28, 1991 and, in revised form, October 18, 1991.

1991 Mathematics Subject Classification. Primary 16A33, 16A35; Secondary 16A03, 16A05.

Key words and phrases. Noetherian rings, embeddings in Artinian rings, sylvester rank functions, filtered rings, graded rings, ore extensions, Morita invariance. 
Definition. A Sylvester rank function for a ring $R$ is a function $\lambda$ : Finitely presented $R$-modules $\rightarrow \frac{1}{n} \mathbb{Z}$ for some $n \in \mathbb{N}$ such that

(i) $\lambda(R)=1$;

(ii) $\lambda(M \oplus N)=\lambda(M)+\lambda(N)$;

(iii) $\lambda\left(M^{\prime \prime}\right) \leq \lambda(M) \leq \lambda\left(M^{\prime}\right)+\lambda\left(M^{\prime \prime}\right)$ for any exact sequence $M^{\prime} \rightarrow M \rightarrow$ $M^{\prime \prime} \rightarrow 0$ of finitely presented $R$-modules.

$\lambda$ is called exact if it is additive over short exact sequences of finitely generated modules.

The fundamental theorem concerning Sylvester rank functions is

Theorem 2.1 (Schofield [15]). Let $R$ be a k-algebra. Then any Sylvester rank function $\lambda$ for $R$ arises from a homomorphism $f: R \rightarrow M_{n}(D), D$ a division ring, in such a way that

(i) $\operatorname{Ker} f=\{a \in R \mid \lambda(R / a R)=1\}$ and

(ii) $\lambda$ is exact if and only if $M_{n}(D)$ is flat as a left $R$-module.

Conversely, a homomorphism $f: R \rightarrow M_{n}(D)$ gives rise to the Sylvester rank function defined by $\lambda_{f}\left(M_{R}\right)=\frac{1}{n}$ length $_{M_{n}(D)} M \otimes_{R} M_{n}(D)$.

In fact, this establishes a one-to-one correspondance between the set of classes of homomorphisms inducing the same Sylvester rank function and the set of Sylvester rank functions.

Part (ii) of Theorem 2.1 motivates the following

Definition. A $k$-algebra $R$ is said to have an exact embedding (or flat embedding) if $R$ admits an exact Sylvester rank function with associated kernel 0 .

We will be primarily interested in the exact embeddability of right Noetherian rings, for which we have available the reduced rank function, $\rho$. For basic results concerning $\rho$ we refer to [13]. In particular, we have that for a right Noetherian ring $R$, the function $\lambda$ defined by $\lambda(M)=\rho(M) / \rho(R)$ is in fact an exact Sylvester rank function. If in addition $R$ is a $k$-algebra then, by Theorem 2.1, $\lambda$ induces a homomorphism from $R$ to a simple Artinian ring. As noted in [3, Theorem 1], the kernel of this homomorphism is precisely the object described in the next

Definition. Let $R$ be a right Noetherian ring. We define the right Sylvester kernel of $R$ to be

$$
K(R)=\left\{a \in R \mid \forall r \in R, \exists c \in \mathscr{C}_{R}(N(R)) \text { such that } \operatorname{arc}=0\right\} .
$$

Furthermore, it has been observed by Lenagan (unpublished) and Krause [12] that $K(R)$ is the unique smallest kernel of an exact Sylvester rank function. We assemble these facts as

Theorem 2.2. Let $R$ be a right Noetherian $k$-algebra. Then $R / K(R)$ has an exact embedding into a simple Artinian ring. Furthermore, if $R$ admits an exact Sylvester rank function with corresponding kernel I (as in Theorem 2.1(i)), then $K(R) \subseteq I$.

Remark. Let $R$ be a right Noetherian ring and $I \triangleleft R$ an ideal of $R$ such that $I \subseteq N(R)$. If $\pi: R \rightarrow R / I$ denotes the canonical projection, then $\pi(K(R)) \subseteq$ $K(R / I)$. 
A commutative Noetherian ring always embeds in an Artinian ring; simply do a primary decomposition of 0 and embed the primary components. Also, by Small's Theorem, $R$ (still commutative Noetherian) has an Artinian quotient ring if and only if $K(R)=0$.

For noncommutative rings the three concepts embedding, exact embedding and having an Artinian ring of quotients are distinct (and, of course, increasingly stronger). The Noetherian $k$-algebra

$$
R=\left(\begin{array}{cc}
k[x] & k[x] /(x) \\
0 & k[x] /(x)
\end{array}\right)
$$

for example, has $K(R)=0$, but does not have an Artinian quotient ring. Furthermore, $R$ has left Sylvester kernel $\left(\begin{array}{cc}0 & k[x] /(x) \\ 0 & 0\end{array}\right)$, so in general left and right Sylvester kernels are distinct for Noetherian rings.

The following is a useful criterion for determining when the Sylvester kernel is 0 .

Theorem 2.3. Let $R$ be a right Noetherian ring such that for any ideal $L \triangleleft R$, $\mathrm{r} . \operatorname{ann}_{R}(L)$ is the right annihilator of some finite subset of $L$. Then

$$
K(R)=0 \Longleftrightarrow \text { All right annihilator prime ideals are minimal. }
$$

Proof. $\Longrightarrow$ Suppose $L P=0$ for some $0 \neq L \triangleleft R$ and a nonminimal prime $P \in \operatorname{Spec}(R)$. By a standard result $P \cap \mathscr{C}_{R}(N(R)) \neq \varnothing$ so $0 \neq L \subseteq K(R)$.

$\Longleftarrow$ Assume $K(R) \neq 0$. Let $P$ be a maximal element in the set of right annihilators of nonzero right ideals contained in $K(R) . P$ is a prime ideal. Let $L=1 . \operatorname{ann}_{R}(P) \cap K(R)$. By the assumption on annihilators we have $P=\mathrm{r} . \operatorname{ann}\left(\left\{x_{1}, \ldots, x_{n}\right\}\right)$ for some $x_{1}, \ldots, x_{n} \in L$. An easy inductive procedure (just as in the proof of [3, Theorem 3]) produces a $c \in \mathscr{C}_{R}(N(R)) \cap P$ whence $P$ is not a minimal prime.

We note that $R$ satisfies the above condition on right annihilators if either $R$ has DCC on right annihilators or $R$ satisfies Gabriel's H-condition, see [13, 6.10.4].

\section{GoING UP, GOING DOWN AND Morita INVARIANCE}

We begin by noting that the proof of [3, Theorem 5] also covers exact embeddability.

Theorem 3.1. If $R$ is a $k$-algebra that is (exactly) embeddable in a simple Artinian ring and if $S$ is a $k$-algebra extension of $R$ that is a finitely generated submodule of a finitely generated free $R$-module, then $S$ is also (exactly) embeddable in a simple Artinian ring.

Going in the opposite direction we have

Theorem 3.2. Suppose that $S$ is a right Noetherian k-algebra. If $R$ is a right Noetherian $k$-subalgebra of $S$ such that $S$ is flat as a left $R$-module, then $K(R) \subseteq K(S) \cap R$. In particular $K(S)=0 \Rightarrow K(R)=0$.

Proof. By Theorem 2.2, $S / K(S)$ embeds in a simple Artinian ring $T$ such that $T$ is flat as a left $S$-module. Consequently $T$ is flat as a left $R$-module so, by Theorem 2.1, $R$ admits an exact Sylvester rank function for which the 
associated kernel is $K(S) \cap R$. It now follows from Theorem 2.2 that $K(R) \subseteq$ $K(S) \cap R$.

In case our flat extension is actually a localization, we have a more explicit relationship between the respective kernels.

Proposition 3.3. If $R$ is a right Noetherian ring and $\mathscr{S}$ is a right Ore set of regular elements of $R$, then $K\left(R_{\mathscr{S}}\right)=K(R) R_{\mathscr{S}}$. In particular $K(R)=0$ if and only if $K\left(R_{\mathscr{S}}\right)=0$.

Proof. Since $P \cap \mathscr{S}=\varnothing$ for any minimal prime $P$ of $R$, we have $N\left(R_{\mathscr{S}}\right)=$ $N(R) R_{\mathscr{S}}$ and so $\mathscr{C}_{R_{\mathscr{S}}}\left(N\left(R_{\mathscr{S}}\right)\right)=\left\{c s^{-1} \in R_{\mathscr{S}} \mid c \in \mathscr{C}_{R}(N(R))\right.$ and $\left.s \in \mathscr{S}\right\}$. Now a straightforward computation using the right Ore condition gives the result.

We now turn to Morita contexts. For definitions and basic results we refer to [13]. We do note, however, those results that will be fundamental in what follows.

Lemma 3.4. If $\left(\begin{array}{ll}R & V \\ W & S\end{array}\right)$ is a Morita context, then there is a one-to-one order preserving correspondence between the lattice of primes of $R$ not containing $V W$ and the lattice of primes of $S$ not containing $W V$. The correspondence is given by $P \leftrightarrow\{s \in S \mid V s W \subseteq P\}$. Furthermore, if this context is actually the ring of a Morita equivalence, then there is a one-to-one order preserving correspondence between the lattice of all ideals of $R$ and the lattice of all ideals of $S$, given by $I \leftrightarrow W I V$, and the rings $R / I$ and $S / W I V$ are again Morita equivalent.

Proposition 3.5. Suppose that $\left(\begin{array}{ll}R & V \\ W & S\end{array}\right)$ is a Morita context, where $R$ and $S$ are right Noetherian rings. Suppose further that $K(R)=0$. If the following conditions are met, then we will also have $K(S)=0$.

(i) $S$ has DCC on right annihilators or satisfies Gabriel's $H$-condition;

(ii) $V W$ is not contained in any minimal prime of $R$;

(iii) $V s W=0$ implies $s=0$.

Proof. Suppose $K(S) \neq 0$. By (i) and Theorem 2.3 we can find a nonminimal prime ideal $P$ in $S$ and a nonzero ideal $L$ such that $L P=0$.

We claim that $V P W$ is not contained in any minimal prime ideal of $R$. Indeed, suppose $V P W \subseteq Q$, where $Q$ is a minimal prime ideal of $R$. By (ii) $V W \nsubseteq Q$ so by the correspondence in Lemma 3.4 we have $Q=\{r \in R \mid W r V \subseteq$ $\left.P^{\prime}\right\}$, where $P^{\prime}$ is a prime ideal of $S$ such that $W V \nsubseteq P^{\prime}$. Furthermore, since all prime ideals contained in $P^{\prime}$ are part of the correspondence we have that $P^{\prime}$ is a minimal prime ideal. Now note that $(W V) P(W V)=W(V P W) V \subseteq$ $W Q V \subseteq P^{\prime}$. Since $P^{\prime}$ is a prime ideal and $W V \nsubseteq P^{\prime}$, we must have $P \subseteq P^{\prime}$, contradicting that $P$ is nonminimal.

Hence $\mathscr{C}_{R}(N(R)) \cap V P W \neq \varnothing$. By (iii) $V L W \neq 0$, and since $V L W \cdot V P W \subseteq$ $V L P W=0$, we conclude that $0 \neq V L W \subseteq K(R)$.

Corollary 3.6. If $R$ is a right Noetherian ring and $e$ is an idempotent of $R$ that is not contained in any minimal prime ideal of $R$, then $K(R)=0$ implies $K(e \operatorname{Re})=0$.

Proof. $e R e$ has DCC on right annihilators since $R$ does. Hence the Morita context $\left(\begin{array}{cc}R & R e \\ e R & e R e\end{array}\right)$ satisfies the hypotheses of Proposition 3.5. 
We now give the main result of this section.

Theorem 3.7. If $R$ and $S$ are right Noetherian rings that are Morita equivalent, then $K(R)$ and $K(S)$ correspond in the sense of Lemma 3.4. In particular, exact embeddability is a Morita invariant for right Noetherian $k$-algebras.

Proof. Let $\left(\begin{array}{ll}R & V \\ W & S\end{array}\right)$ be the context of the equivalence. We must show that $K(S)=W K(R) V$. By Lemma 3.4, $R / K(R)$ and $S / W K(R) V$ are again Morita equivalent rings, and since $R / K(R)$ has DCC on right annihilators (Theorem 2.2), it follows that $S / W K(R) V$ also has DCC on right annihilators. The remaining conditions in Proposition 3.5 are trivially satisfied by the Morita context giving the equivalence of $R / K(R)$ and $S / W K(R) V$, so we conclude that $K(S / W K(R) V)=0$. Since $K(R) \subseteq N(R)$ implies that $W K(R) V \subseteq N(S)$, an application of the remark after Theorem 2.2 shows that $K(S) \subseteq W K(R) V$. A symmetric argument yields $K(R) \subseteq V K(S) W$, so $K(S) \subseteq W K(R) V \subseteq W V K(S) W V=K(S)$. This forces $K(S)=W K(R) V$, as required.

Unlike the situation for embeddings in Artinian rings or having Artinian quotient rings, the exact embeddability of $R$ does not guarantee the exact embeddability of $e R e$ for an arbitrary idempotent $e \in R$.

Proposition 3.8. Suppose that $R$ is a right Noetherian ring that may be embedded in an Artinian ring. Then there exists a right Noetherian ring $S$ such that $K(S)=0$ and $R \simeq e$ Se for some idempotent $e \in S$.

Proof. Suppose that $R$ embeds in the Artinian ring $T$, and set $S=\left(\begin{array}{ll}R & T \\ 0 & T\end{array}\right)$. Any nonminimal prime ideal of $S$ is of the form $\left(\begin{array}{ll}P & T \\ 0 & T\end{array}\right)$, where $P$ is a nonminimal prime of $R$. Clearly no such ideal can have a nonzero left annihilator. Since $S$ inherits DCC on right annihilators from $M_{2}(T)$, we can apply Theorem 2.3 to conclude that $K(S)=0$.

For example, every commutative Noetherian $k$-algebra may be realized in this way as a "corner" of a ring having an exact embedding.

\section{ORE EXTENSIONS AND GRADED RINGS}

We now study the Sylvester kernels of Ore extensions and graded rings.

Lemma 4.1. Let $R$ be a right Noetherian ring, $\sigma \in \operatorname{Aut}(R)$, and $\delta$ a $\sigma$ derivation of $R$. Assume that $\delta(N(R)) \subseteq N(R)$. Then for all

$$
c \in \mathscr{C}_{R[x ; \sigma, \delta]}(N(R[x ; \sigma, \delta])),
$$

there exists an $f \in R[x ; \sigma, \delta]$ and an $n \in N(R[x ; \sigma, \delta])$ such that $c f=$ $\sum_{i=0}^{m} d_{i} x^{i}+n$, where $d_{m} \in \mathscr{C}_{R}(N(R))$.

Proof. A straightforward modification of [16, Lemma 2].

Theorem 4.2. Let $R$ be a right Noetherian ring, $\sigma \in \operatorname{Aut}(R)$, and $\delta$ a $\sigma$ derivation. If $\delta(N(R)) \subseteq N(R)$, then $K(R[x ; \sigma, \delta])=K(R) R[x ; \sigma, \delta]$, and in particular $K(R)=0$ if and and only if $K(R[x ; \sigma, \delta])=0$.

Proof. First consider $f=f_{0}+f_{1} x+\cdots+f_{n} x^{n} \in K(R) R[x ; \sigma, \delta]$. Since $\mathscr{C}_{R}(N(R))$ is $\sigma$-invariant we can find a $c \in \mathscr{C}_{R}(N(R))$ such that $f_{n} x^{n} c=$ 
$f_{n}\left(g_{0}+g_{1} x+\cdots+g_{n-1} x^{n-1}\right)$ for some $g_{0}, \ldots, g_{n-1} \in R$. Consequently $f_{c} \in K(R) R[x ; \sigma, \delta]$ and the degree of $f c$ is at most $n-1$. Since $\mathscr{C}_{R}(N(R))$ is multiplicatively closed and contained in $\mathscr{C}_{R[x ; \sigma, \delta]}(N(R[x ; \sigma, \delta])$, an inductive procedure shows that $K(R) R[x ; \sigma, \delta] \subseteq K(R[x ; \sigma, \delta])$.

Conversely, set $\mathscr{D}=\left\{\sum_{i=0}^{n} d_{i} x^{i} \mid n \in \mathbb{N} \cup\{0\} ; d_{n} \in \mathscr{C}_{R}(N(R))\right\}$. Note again that $\mathscr{C}_{R}(N(R))$ is $\sigma$-invariant, so $\mathscr{D}$ is multiplicatively closed. Let $k=k_{0}+$ $\cdots+k_{m} x^{m} \in K(R[x ; \sigma, \delta])$. We can find $c \in \mathscr{C}_{R[x ; \sigma, \delta]}(N(R[x ; \sigma, \delta]))$ such that $k c=0$. Applying Lemma 4.1 to $c$, we see that there exists a $d \in \mathscr{D}$ and an $n \in N(R[x ; \sigma, \delta])$ such that $k d=k n$. Now apply this to $k d$; we get $k d d^{\prime}=k n n^{\prime}$. Since $\mathscr{D}$ is multiplicatively closed and $N(R[x ; \sigma, \delta])$ is nilpotent we get that for all $r \in R$ we can find $d \in \mathscr{D}$ such that $k r d=0$. We conclude that $k_{m} \in K(R)$, and hence, by the first part of the proof, that $k_{0}+\cdots+k_{n-1} x^{n-1} \in K(R[x ; \sigma, \delta])$. An easy induction on $n$ now yields $K(R[x ; \sigma, \delta]) \subseteq K(R) R[x ; \sigma, \delta]$.

In $[3,4]$ it is proved that $R[x ; \sigma]$ embeds in an Artinian ring if either $R$ embeds in an Artinian ring and $\sigma$ has finite order or $R$ is commutative Noetherian, and it was conjectured that the result holds if $R$ is just assumed to be embeddable in an Artinian ring. Theorem 4.2 settles this question in the affirmative for exact embeddability.

Next we consider group graded rings. For notation and basic results on graded rings we refer to [5].

Proposition 4.3. Let $A=\bigoplus_{g \in G} A_{g}$ be strongly graded by the finite group $G$. If $A_{e}$ is a right Noetherian k-algebra then $K\left(A_{e}\right)=0$ if and only if $K(A)=0$ if and only if $K\left(A \# k[G]^{*}\right)=0$.

Proof. This follows from Theorems 3.1 and 3.2, since ${ }_{A_{e}} A$ and ${ }_{A} A \# k[G]^{*}$ are finitely generated and projective.

Proposition 4.4. Let $A=\bigoplus_{n \in \mathbb{Z}} A_{n}$ be strongly graded and $A_{0}$ a right Noetherian ring. Then $K(A)=K\left(A_{0}\right) A$, and so in particular $K\left(A_{0}\right)=0$ if and only if $K(A)=0$.

Proof. By [9, Lemma 3.3] (yet another version of [16, Lemma 2]) we have that for all $f \in \mathscr{C}_{A}(N(A))$ there exists a $g \in A$ such that $f g=d+n$ where $d_{0} \in$ $\mathscr{C}_{A_{0}}\left(N\left(A_{0}\right)\right)$ and $n \in N(A)$. Now the proof is carried out as a straightforward modification of the proof of Theorem 4.2. We omit the details.

A well-known inductive procedure based on Propositions 4.3 and 4.4 yields.

Corollary 4.5. Let $G$ be a poly-cyclic-by-finite group and let $A=\bigoplus_{g \in G} A_{g}$ be a ring strongly graded by $G$. If $A_{e}$ is a right Noetherian $k$-algebra then $K\left(A_{e}\right)=0$ if and only if $K(A)=0$.

\section{Filtered RINGS}

In this section we consider the question of exact embeddings of filtered rings into Artinian rings. Incidentally, our approach yields a new and very different proof of a result on Artinian quotient rings (see [10] and Theorem 5.6 hereafter) in the Noetherian case. 
Let us first fix some notation and recall some basic facts. For proofs we refer to [1]. Let $R$ be a ring with an ascending filtration $\left\{F_{n} R\right\}_{n \in \mathbb{Z}}$, i.e., $F_{n} R \subseteq F_{n+1} R, F_{n} R F_{m} R \subseteq F_{n+m} R$, and $1 \in F_{0} R$. We consider only exhaustive $\left(\bigcup_{n \in \mathbb{Z}} F_{n} R=R\right)$ and separated $\left(\bigcap_{n \in \mathbb{Z}} F_{n} R=0\right)$ filtrations. Recall that the associated graded ring $\operatorname{gr}(R)$ is defined by $\operatorname{gr}(R)=\bigoplus_{n \in \mathbb{Z}} F_{n} R / F_{n-1} R$ and the Rees ring, $\widetilde{R}$, is defined by $\widetilde{R}=\bigoplus_{n \in \mathbb{Z}} F_{n} R$. Letting $X$ be an indeterminate we have the following:

(i) $\widetilde{R} \simeq \sum_{n \in \mathbb{Z}} F_{n} R X^{n} \subseteq R\left[X^{-1}, X\right]$, where the isomorphism (of graded rings) takes the central regular element $1 \in F_{1} R \subseteq \widetilde{R}$ to $X$. Henceforth we identify $\widetilde{R}$ and its image in $R\left[X^{-1}, X\right]$;

(ii) $\operatorname{gr}(R)=\widetilde{R} / X \widetilde{R}$;

(iii) $\widetilde{R}\left[X^{-1}\right]=R\left[X^{-1}, X\right]$;

(iv) If $\operatorname{gr}(R)$ is (right) Noetherian and $F_{-1} R \subseteq J\left(F_{0} R\right)$, then $\widetilde{R}$ is (right) Noetherian.

For the proof of our main result we need the following proposition, which is of some independent interest.

Proposition 5.1. Let $R$ be a Noetherian ring or a right FBN ring, and suppose that $x \in R$ is a regular normal element such that $\bigcap_{i} x^{i} R=0$. If $K(R / x R)=0$ then $K(R)=0$.

Proof. Note that we can apply Theorem 2.3 to $R$, so if $K(R) \neq 0$ there exists a nonminimal prime ideal $P$ of $R$ such that $L=1 \cdot \operatorname{ann}_{R}(P) \neq 0$. Since $x$ is regular, $x \notin P$. Letting $Q_{1}, \ldots, Q_{n}$ be the prime ideals minimal over $x R$ we have, by Jategaonkar's Principal Ideal Theorem (see [8] or [13]), that $\left(P+Q_{i}\right) / Q_{i} \neq 0$ for all $i$.

By a standard argument (Goldie's theorem) it follows that $P \cap \mathscr{C}_{R}\left(\bigcap_{i} Q_{i}\right)$ $\neq \varnothing$. Since $N\left(R / x^{n} R\right)=\bigcap_{i} Q_{i} / x^{n} R$ for all $n \in \mathbb{N}$ we conclude that $\left(L+x^{n} R\right) / x^{n} R \subseteq K\left(R / x^{n} R\right)$ and, by a straightforward computation, $K\left(R / x^{n} R\right)=0$ for all $n \in \mathbb{N}$.

Thus, for all $n \in \mathbb{N}$ we have $L \subseteq x^{n} R$, which implies $L=0$, a contradiction. Hence $K(R)=0$.

We now apply this to filtered rings.

Theorem 5.2. Let $R$ be a ring with a filtration $\left\{F_{n} R\right\}_{n \in \mathbb{Z}}$ such that $F_{-1} R \subseteq$ $J\left(F_{0} R\right)$. If either $\operatorname{gr}(R)$ is Noetherian or $R$ is $P I$ and $\operatorname{gr}(R)$ is right Noetherian, then $K(\operatorname{gr}(R))=0$ implies $K(R)=0$.

Proof. We have the following diagram (recall our notation):

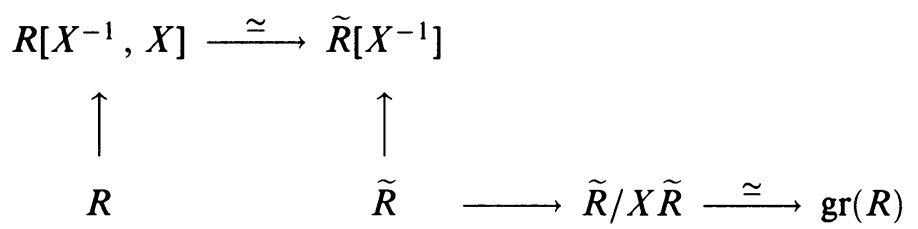

Since $\widetilde{R}$ and $X$ satisfy the hypothesis of Proposition 5.1 we have that $K(\operatorname{gr}(R))=0$ implies $K(\widetilde{R})=0$. By Proposition $3.3 K(\widetilde{R})=0$ if and only if $K\left(\tilde{R}\left[X^{-1}\right]\right)=0$. Finally, $K\left(R\left[X^{-1}, X\right]\right)=0$ if and only if $K(R)=0$ by either Proposition 4.4 or Theorem 4.2 combined with Proposition 3.3. 
Propositions 5.1 and 5.2 apply to a number of situations of which we single out three: PBW-extensions, Ore extensions, and power series rings. Recall that $S=R\left[\theta_{1}, \ldots, \theta_{n}\right]$ is called a PBW-extension of $\mathrm{R}$ if (1) $S_{R}$ is free with basis $\left\{\theta_{1}^{k_{1}} \cdots \theta_{n}^{k_{n}} \mid k_{i} \in \mathbb{N} \cup\{0\}\right\},(2)\left[\theta_{i}, \theta_{j}\right] \in R+\sum_{i} \theta_{i} R$, and (3) $\left[R, \theta_{i}\right] \subseteq R$, see $[2,13,14]$.

Corollary 5.3. Let $S=R\left[\theta_{1}, \ldots, \theta_{n}\right]$ be a PBW-extension of a Noetherian ring $R$. Then $K(R)=0$ implies $K(S)=0$.

Proof. The obvious filtration of $S$ makes $\operatorname{gr}(S)$ a polynomial ring over $R$. Repeated application of Proposition 5.1 yields that $K(\operatorname{gr}(S))=0$, and then an application of Theorem 5.2 yields $K(S)=0$.

As a second application of Theorem 5.2 we note that we can remove the condition on $\delta$ in Theorem 4.2 if we assume that $R$ is Noetherian.

Corollary 5.4. Let $S=R[x ; \sigma, \delta]$ be an Ore extension of a Noetherian ring $R$. Then $K(R)=0$ implies $K(S)=0$.

Proof. The obvious filtration makes $\operatorname{gr}(S)$ a skew polynomial ring over $R$. Now apply Proposition 5.1 (or Corollary 4.3) and Theorem 5.2.

Finally a direct application of Proposition 5.1 yields

Corollary 5.5. Let $R$ be a Noetherian ring. Then $K(R)=0$ implies $K(R[[x]])=$ 0 .

Remark. The extension rings in the above corollaries are all free as modules over the smaller ring. Thus Theorem 3.2 applies to yield converses in case we assume $R$ is a $k$-algebra.

A technique similar to the one used in the proof of Theorem 5.2 yields a result on quotient rings for Noetherian rings. This result is, as mentioned, known even for right Noetherian rings (see [10]), so we only sketch our proof:

Theorem 5.6. Let $R$ be a Noetherian ring and $x \in R$ a normal regular element such that $\bigcap_{i} x^{i} R=0$. Assume that $R / x R$ has an Artinian ring of quotients. Then so does $R$.

Proof. One easily sees that $R / x^{n} R$ has an Artinian quotient ring for all $n$ by checking Small's criterion. As noted in Krause [11], the theorem follows if all middle annihilator prime ideals of $R$ are minimal.

Let $P=\operatorname{Mid}(A, B)$ be a middle annihilator prime ideal, where $A, B \triangleleft R$ and $A B \neq 0$. Since $x$ is normal and regular, $x \notin P$. If $P$ is not minimal it is seen, just as in the proof of Propostion 5.1, that $P \cap C_{R}\left(x^{n} R\right) \neq 0$ for all $n$. Picking $n$ such that $A B \nsubseteq x^{n} R$ we see that

$$
\left(A+x^{n} R\right) / x^{n} R \cdot\left(c+x^{n} R\right) \cdot\left(B+x^{n} R\right) / x^{n} R=0
$$

for some regular $c+x^{n} R$ in $R / x^{n} R$. The fact that $R / x^{n} R$ has a full quotient ring implies $A B \subseteq x^{n} R$, a contradiction. Hence all middle annihilator primes are minimal, and we are done.

Corollary 5.7. Let $R$ be a filtered ring such that $\operatorname{gr}(R)$ is Noetherian and $F_{-1} R \subseteq$ $J\left(F_{0} R\right)$. If $\operatorname{gr}(R)$ has an Artinian ring of quotients, then so does $R$.

Proof. Just as Theorem 5.2 since "having an Artinian ring of quotients" travels the same diagram (see [10]). 


\section{ACKNOWLEDGMENT}

The authors would like to thank L. W. Small for his suggestions and helpful conversations regarding this line of research. The material presented here will also appear in the authors' dissertations.

\section{REFERENCES}

1. M. Asensio, F. van Oystaeyen, and M. Van Den Bergh, A new algebraic approach to microlocalisation of filtered rings, Trans. Amer. Math. Soc. 116 (1989), 537-553.

2. A. D. Bell and K. G. Goodearl, Uniform rank of differential operator rings and Poincaré Birkhoff Witt extensions, Pacific J. Math. 131 (1988), 13-37.

3. W. Blair and L. W. Small, Embeddings in Artinian rings and Sylvester rank functions, Israel J. Math. 58 (1987), 10-18.

4. __ Embedding differential and skew polynomial rings into artinian rings, Proc. Amer. Math. Soc. 109 (1990), 881-886.

5. M. Cohen and S. Montgomery, Group graded rings, smash products and group actions, Trans. Amer. Math. Soc. 282 (1984), 237-258.

6. C. Dean, Nonembeddable factors of enveloping algebras of semi simple Lie algebras, Bull. London Math. Soc. 21 (1989), 65-69.

7. C. Dean and J. T. Stafford, A nonembeddable Noetherian ring, J. Algebra 115 (1988), 175-181.

8. A. V. Jategaonkar, Relative Krull dimension and prime ideals in right Noetherian rings, Comm. Algebra 2 (1974), 429-468.

9. A. Jensen and S. Jøndrup, Classical quotient rings of group graded rings (to appear).

10. A. Jensen, S. Jøndrup and M. Van Den Bergh, Artinian quotient rings of filtered rings (to appear).

11. G. Krause, Middle annihilators in Noetherian rings, Comm. Algebra 8 (1980), 781-791.

12. __, Additive rank functions in Noetherian rings, J. Algebra 130 (1990), 451-461.

13. J. C. McConnell and J. C. Robson, Noncommutative noetherian rings, Wiley Interscience, New York, 1987.

14. D. S. Passman, Prime ideals in enveloping rings, Trans. Amer. Math. Soc. 302 (1987), 535-560.

15. A. Schofield, Representations of rings over skew fields, London Math. Soc. Lecture Note Ser., vol. 92, Cambridge Univ. Press, Cambridge, 1985.

16. L. W. Small, Orders in artinian rings II, J. Algebra 9 (1968), 266-273.

17. 147-148.

Department of Mathematics, University of California, San Diego, la Jolla, CaliforNIA 92093

E-mail address: Beaulieu@euclid.ucsd.edu

E-mail address: AndersJ@math.ku.dk 